\title{
On the estimation of the domain of attraction for discrete-time switched and hybrid nonlinear systems
}

\author{
C. K. Luk and G. Chesi \\ Department of Electrical and Electronic Engineering \\ The University of Hong Kong \\ Email: chuenkit@eee.hku.hk
}

\begin{abstract}
This paper addresses the estimation of the domain of attraction for discrete-time nonlinear systems where the vector field is subject to changes. Firstly, the paper considers the case of switched systems, where the vector field is allowed to arbitrarily switch among the elements of a finite family. Secondly, the paper considers the case of hybrid systems, where the state space is partitioned into several regions described by polynomial inequalities, and the vector field is defined on each region independently from the other ones. In both cases, the problem consists of computing the largest sublevel set of a Lyapunov function included in the domain of attraction. An approach is proposed for solving this problem based on convex programming, which provides a guaranteed inner estimate of the sought sublevel set. The conservatism of the provided estimate can be decreased by increasing the size of the optimization problem. Some numerical examples illustrate the proposed approach.
\end{abstract}

\section{Introduction}

A key problem in control engineering consists of determining the domain of attraction of an equilibrium point. In fact, this is important to avoid system failures. Classical applications include pendulum systems, tunnel diode circuits, mass-spring systems, and negative-resistance oscillators ([11]). More recently, applications have been found in other fields such as biology, in order to search for an optimal strategy for cancer treatment by analyzing the tumor growth dynamics ([13]), and ecology, in order to investigate the resilience of an ecological system $([12])$. 
Determining the domain of attraction of an equilibrium point of a nonlinear dynamical system is a challenging problem. Indeed, it amounts to determining all the initial conditions for which the solution of a system of differential equations (in the case of continuous-time systems) or difference equations (in the case of discrete-time systems) eventually converges to the considered equilibrium point. Unfortunately, this cannot be done exactly, either analytically or numerically, except for few very special cases. Among the numerous methods that have been proposed in the literature for estimating the domain of attraction, one should recall the classical Zubov method, La Salle method, the trajectory reversing method, and the use of maximal Lyapunov functions $([9,20])$. Some recent methods have exploited sum-of-squares (SOS) polynomials to provide estimates of the domain of attraction, such as $\underline{([8,18,10,7])}$ which consider polynomial systems, $(\underline{\underline{[19}, \underline{6]})}$ which consider uncertain polynomial systems, and ([3]) which consider nonpolynomial systems. See also $([5,15,16])$ and references therein.

Switched and hybrid nonlinear systems are two very important classes of dynamical systems for control engineering, see for example ([2]) and references therein. In fact, real systems are nowadays more and more complex, presenting piecewise dynamics. However, there exist only few contributions addressing the estimation of the domain of attraction for these classes of dynamical systems. Such contributions consider the case of continuous-time dynamical systems, such as ([14]), which investigates the use of maximal Lyapunov functions, and ([1]), which considers a special class of hybrid systems that exhibit jumps in the state trajectory. For discrete-time switched and hybrid systems, the estimation of the domain of attraction has not been addressed yet in the literature.

This paper addresses the estimation of the domain of attraction for discrete-time nonlinear systems where the vector field is subject to changes. Firstly, the paper considers the case of switched systems, where the vector field is allowed to arbitrarily switch among the elements of a finite family. Secondly, the paper considers the case of hybrid systems, where the state space is partitioned into several regions described by polynomial inequalities, and the vector field is defined on each region independently from the other ones. In both cases, the problem consists of computing the largest sublevel set of a Lyapunov function included in the domain of attraction. An approach is proposed for solving this problem based on convex programming, which provides a guaranteed inner estimate of the sought sublevel set. The conservatism of the provided estimate can be decreased by increasing the size of the optimization problem. Some numerical examples illustrate the proposed approach. 
This paper is organized as follows. Section 2 introduces the problem formulation and some preliminaries about sum-of-squares (SOS) polynomials. Section 3 describes the proposed approach. Section 4 presents the illustrative examples. Section 5 reports the conclusion.

\section{Preliminaries}

\subsection{Problem formulation}

Notation:

- $\mathbb{R}$ : Space of real numbers;

- $0_{n}: n \times 1$ null vector;

- $A^{\prime}$ : Transpose of matrix $A$;

- $A>0(A \geq 0)$ : positive definite (semidefinite) matrix;

- s.t.: subject to.

Consider a discrete-time hybrid nonlinear systems in the form

$$
x(t+1)=f(x(t))
$$

where $x \in \mathbb{R}^{n}$ is the state and $f: \mathbb{R}^{n} \rightarrow \mathbb{R}^{n}$.

The domain of attraction of the origin of (1) is the set of initial conditions for which the system converges to the origin, i.e.

$$
\mathcal{D}=\left\{x_{0} \in \mathbb{R}^{n}: \lim _{t \rightarrow+\infty} x(t)=0_{n}\right\}
$$

where $x_{0}$ is the initial condition of (1).

Let $v: \mathbb{R}^{n} \rightarrow \mathbb{R}$ be a radially unbounded positive definite function. We say that $v(x)$ is a Lyapunov function for system (1) if

$$
\exists \delta>0: \Delta v(x)<0 \forall x: 0<\|x\|<\delta
$$

where

$$
\begin{aligned}
\Delta v(x) & =v(x(t+1))-v(x(t)) \\
& =v(f(x(t)))-v(x(t))
\end{aligned}
$$

The sublevel set $\mathcal{V}(c)$ of $v(x)$ is defined as

$$
\mathcal{V}(c)=\left\{x \in \mathbb{R}^{n}: v(x) \leq c\right\} .
$$


We have that $\mathcal{V}(c)$ is an estimate of $\mathcal{D}$ if

$$
\Delta v(x)<0 \forall x \in \mathcal{V}(c) \backslash\left\{0_{n}\right\} .
$$

The largest estimate $\mathcal{V}\left(c^{*}\right)$ of $\mathcal{D}$ can be written as

$$
c^{*}=\sup \{c \in \mathbb{R}: \text { (6) holds }\} .
$$

We consider the following two problems.

Problem A: Compute the largest estimate $\mathcal{V}\left(c^{*}\right)$ of $\mathcal{D}$ in the case of switched systems, i.e.

$$
f(x) \in \bigcup_{i=1}^{N} f_{i}(x)
$$

where $f_{i}: \mathbb{R}^{n} \rightarrow \mathbb{R}^{n}, i=1, \ldots, N$, are polynomial functions, that is, $f(x)$ is allowed to arbitrarily switch among $f_{1}(x), \ldots, f_{N}(x)$.

Problem B: Compute the largest estimate $\mathcal{V}\left(c^{*}\right)$ of $\mathcal{D}$ in the case of hybrid systems, i.e.

$$
f(x)=f_{i}(x) \text { if } x \in \mathcal{X}_{i} i=1, \ldots, N
$$

where $f_{i}: \mathbb{R}^{n} \rightarrow \mathbb{R}^{n}, i=1, \ldots, N$, are polynomial functions, and $\mathcal{X}_{i} \subseteq \mathbb{R}^{n}$ is defined by

$$
\begin{aligned}
\mathcal{X}_{i} & =\left\{x \in \mathbb{R}^{n}: z_{i}(x) \geq 0\right\}, i=1, \ldots, N-1 \\
\mathcal{X}_{N} & =\mathbb{R}^{n} \backslash \mathcal{X}_{1} \backslash \ldots \backslash \mathcal{X}_{N-1}
\end{aligned}
$$

where $z_{i}: \mathbb{R}^{n} \rightarrow \mathbb{R}, i=1, \ldots, N-1$, are polynomials. We assume that

$$
\mathcal{X}_{i} \cap \mathcal{X}_{j}=\emptyset \text { if } i \neq j
$$

that means there are no overlaps among the regions $\mathcal{X}_{1}, \ldots, \mathcal{X}_{N}$. We also assume that $f\left(0_{n}\right)=0_{n}$, and that the origin is the equilibrium point of interest in both problems.

\subsection{SOS polynomials}

Here we report some preliminaries about SOS polynomials. Let $v: \mathbb{R}^{n} \rightarrow \mathbb{R}$ be a polynomial of degree not greater than $2 m$. Then, $v(x)$ can be expressed as

$$
v(x)=x^{\{m\}^{\prime}}(V+L(\alpha)) x^{\{m\}},
$$


where $x^{\{m\}}$ is a vector containing all the monomials of degree less than or equal to $m$ in $x, V$ is a symmetric matrix such that

$$
v=x^{\{m\}^{\prime}} V x^{\{m\}},
$$

$L(\alpha)$ is a linear parametrization of

$$
\left\{L=L^{\prime}: x^{\{m\}^{\prime}} L x^{\{m\}}=0\right\},
$$

and $\alpha$ is a free vector.

This representation is called Gram matrix method and square matricial representation (SMR). It turns out that $v(x)$ is a SOS polynomial if and only if there exists $\alpha$ such that

$$
V+L(\alpha) \geq 0
$$

which is a linear matrix inequality (LMI). See ([4]) and references therein for details.

\section{Proposed Approach}

Let us consider Problem A. For $i=1, \ldots, N$, let us define

$$
\begin{aligned}
c_{i}^{*}= & \sup c \\
& \text { s.t. } \Delta v_{i}(x)<0 \quad \forall x \in \mathcal{V}(c) \backslash\left\{0_{n}\right\} .
\end{aligned}
$$

From (7) it follows that

$$
c^{*}=\min \left\{c_{1}^{*}, \ldots, c_{N}^{*}\right\} .
$$

The following result provides a condition for the estimation of the domain of attraction in Problem A.

Theorem 1 Let us consider the system (1) and (8). Let $v: \mathbb{R}^{n} \rightarrow \mathbb{R}$ be a positive definite polynomial. Let $c$ be a positive real number. Suppose that, for all $i=1, \ldots, N$, there exist polynomials $q_{i}: \mathbb{R}^{n} \rightarrow \mathbb{R}$ and $\varepsilon>0$ such that

$$
\left\{\begin{array}{l}
-\Delta v_{i}(x)-q_{i}(x)(c-v(x))-\varepsilon\|x\|^{2} \text { is } S O S \\
q_{i}(x) \text { is } S O S
\end{array}\right.
$$

where

$$
\Delta v_{i}(x)=v\left(f_{i}(x(t))\right)-v(x(t)) .
$$

Then, $v(x)$ is a Lyapunov function for the origin, and $\mathcal{V}(c) \subseteq \mathcal{D}$. 
Proof. Let us suppose that there exist polynomials $q_{i}(x)$ and $\varepsilon>0$ such that (18) holds. Let us consider any $x$ in the set $\mathcal{V}(c) \backslash\left\{0_{n}\right\}$. From the second condition in (18), it follows that

$$
\begin{aligned}
q_{i}(x) & =\sum_{j=1}^{k} q_{i, j}(x)^{2} \\
& \geq 0
\end{aligned}
$$

for some polynomials $q_{i, j}(x)$. This condition, the first condition in (18), and $v(x) \leq c$, imply that

$$
\begin{aligned}
0 & \leq-\Delta v_{i}(x)-q_{i}(x)(c-v(x))-\varepsilon\|x\|^{2} \\
& \leq-\Delta v_{i}(x)-\varepsilon\|x\|^{2}
\end{aligned}
$$

Since $\varepsilon>0$ and $x \neq 0$, it follows that

$$
\Delta v_{i}(x)<0 .
$$

From $(3), v(x)$ is a Lyapunov function for the origin of the system. Moreover, from (6), we can conclude that $\mathcal{V}(c) \subseteq \mathcal{D}$.

Theorem 1 provides a condition for establishing whether $\mathcal{V}(c)$ is an inner estimate of $\mathcal{D}$ in Problem A. For $i=1, \ldots, N$, let us define

$$
\begin{aligned}
\hat{c}_{i}= & \sup c \\
& \text { s.t. } \exists q_{i}(x), s_{i}(x): \text { (18) holds. }
\end{aligned}
$$

Hence, the best lower bound guaranteed by Theorem 1 for Problem A is

$$
\hat{c}=\min \left\{\hat{c}_{1}, \ldots, \hat{c}_{N}\right\} .
$$

Let us consider Problem B. For $i=1, \ldots, N$, let us define

$$
\begin{aligned}
c_{i}^{*}= & \sup c \\
& \text { s.t. } \Delta v_{i}(x)<0 \quad \forall x \in \mathcal{X}_{i} \cap \mathcal{V}(c) \backslash\left\{0_{n}\right\} .
\end{aligned}
$$

With these values of $c_{i}^{*}$, let us define $c^{*}$ as in (17). The following result provides a condition for the estimation of the domain of attraction in Problem B.

Theorem 2 Let us consider the system (1) and (9). Let $v: \mathbb{R}^{n} \rightarrow \mathbb{R}$ be a positive definite polynomial. Let $c$ be a positive real number. Suppose 
that, for all $i=1, \ldots, N$ and $j=1, \ldots, N-1$, there exist polynomials $q_{i}: \mathbb{R}^{n} \rightarrow \mathbb{R}, s_{i}: \mathbb{R}^{n} \rightarrow \mathbb{R}, t_{j}: \mathbb{R}^{n} \rightarrow \mathbb{R}$ and $\varepsilon>0$ such that

$$
\text { if } i<N,\left\{\begin{array}{l}
-\Delta v_{i}(x)-q_{i}(x)(c-v(x))-s_{i}(x) z_{i}(x)-\varepsilon\|x\|^{2} \text { is } S O S \\
q_{i}(x) \text { is } S O S \\
s_{i}(x) \text { is } S O S
\end{array}\right.
$$

and

if $i=N,\left\{\begin{array}{l}-\Delta v_{N}(x)-q_{N}(x)(c-v(x))+\sum_{j=1}^{N-1} t_{j}(x) z_{j}(x)-\varepsilon\|x\|^{2} \text { is } S O S \\ q_{N}(x) \text { is } S O S \\ t_{j}(x) \text { is } S O S\end{array}\right.$

where $\Delta v_{i}(x)$ is as in (19). Then, $v(x)$ is a Lyapunov function for the origin of the system, and $\mathcal{V}(c) \subseteq \mathcal{D}$.

Proof. Let us suppose that there exist polynomials $q_{i}(x), s_{i}(x)$ and $t_{j}(x)$, and $\varepsilon>0$ such that (23)-(24) hold. Let us consider any $x$ in the set $\mathcal{V}(c) \backslash\left\{0_{n}\right\}$. From the last two conditions in (23) and (24), we have

$$
q_{i}(x) \geq 0, s_{i}(x) \geq 0, t_{j}(x) \geq 0 .
$$

If $x \in \mathcal{X}_{i}$ for some $i$ with $i<N$, let us consider (23) for such a value of $i$. It follows that

$$
\begin{aligned}
0 & \leq-\Delta v_{i}(x)-q_{i}(x)(c-v(x))-s_{i}(x) z_{i}(x)-\varepsilon\|x\|^{2} \\
& <-\Delta v_{i}(x)
\end{aligned}
$$

since $q_{i}(x) \geq 0, c-v(x) \geq 0, s_{i}(x) \geq 0, z_{i}(x) \geq 0, \varepsilon>0$ and $x \neq 0$. Otherwise, one has that $x \in \mathcal{X}_{N}$, and from (24) it follows that

$$
\begin{aligned}
0 & \leq-\Delta v_{N}(x)-q_{N}(x)(c-v(x))+\sum_{j=1}^{N-1} t_{j}(x) z_{j}(x)-\varepsilon\|x\|^{2} \\
& <-\Delta v_{i}(x)
\end{aligned}
$$

since $q_{N}(x) \geq 0, c-v(x) \geq 0, t_{j}(x) \geq 0, z_{j}(x)<0, \varepsilon>0$ and $x \neq 0$. From (3), $v(x)$ is a Lyapunov function for the origin of the system. Moreover, from (6), we can conclude that $\mathcal{V}(c) \subseteq \mathcal{D}$.

Theorem 2 provides a condition for establishing whether $\mathcal{V}(c)$ is included in $\mathcal{D}$ in Problem B. For $i=1, \ldots, N-1$, let us define

$$
\begin{aligned}
\hat{c}_{i}= & \sup c \\
& \text { s.t. } \exists q_{i}(x), s_{i}(x): \text { (23) holds, }
\end{aligned}
$$


and, for $i=N$, let us define

$$
\begin{aligned}
\hat{c}_{N}= & \sup c \\
& \text { s.t. } \exists q_{N}(x), t_{j}(x):(24) \text { holds. }
\end{aligned}
$$

Hence, the best lower bound guaranteed by Theorem 2 for Problem B is $\hat{c}$ in (21) with $\hat{c}_{1}, \ldots, \hat{c}_{N}$ given by $(25)-(26)$.

\section{Examples}

In the following examples, the conditions of Theorem 1 and 2 are checked in MATLAB with the toolbox SeDuMi ([17]) by transforming the SOS conditions into LMIs as explained in Section 2.2. Before proceeding it is worth noticing that the main conservatism of the estimates $V(c)$ provided by Theorems 1 and 2 comes from the fact that the Lyapunov function $v(x)$ is fixed. Less conservative estimates can be obtained by searching for $v(x)$ at the expenses of making the problem nonconvex.

\subsection{Example 1}

Consider a discrete-time nonlinear hybrid system in the form of (1) with

$f_{1}(x)=\left(\begin{array}{c}0.5 x_{1} \\ -0.8 x_{2}-x_{1}^{2}\end{array}\right)$,

$f_{2}(x)=\left(\begin{array}{c}0.5 x_{1}+x_{1} x_{2} \\ -0.8 x_{2}\end{array}\right)$,

with the function $v=x_{1}^{2}+x_{2}^{2}$.

Let us consider Problem A (switched system). Figure 1 and Figure 2 show the largest estimates of the domain of attraction of individual subsystems $f_{1}(x)$ and $f_{2}(x), \mathcal{V}\left(\hat{c}_{1}\right)$ and $\mathcal{V}\left(\hat{c}_{2}\right)$ respectively. By using Theorem 1 , computational results show that $\hat{c}_{1}=0.4469$ and $\hat{c}_{2}=0.8866$. Hence, $\hat{c}=\min \left\{\hat{c}_{1}, \hat{c}_{2}\right\}=0.4469$. As guaranteed by Theorem $1, \mathcal{V}(\hat{c})$ is a guaranteed inner estimate of $\mathcal{D}$.

Next, let us consider Problem B (hybrid system). Consider $z_{1}(x)=x_{2}$, we have

$\mathcal{X}_{1}=\left\{x: z_{1}(x) \geq 0\right\}$, 


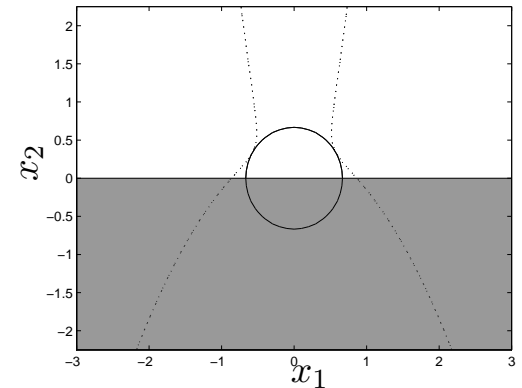

Figure 1: Example 4.1: $\mathcal{X}_{1}$ (white area), the curve $\Delta v_{1}(x)=0$ (dotted lines), the curve $\mathcal{V}\left(\hat{c}_{1}\right)$ for both Problem A and B (solid line).

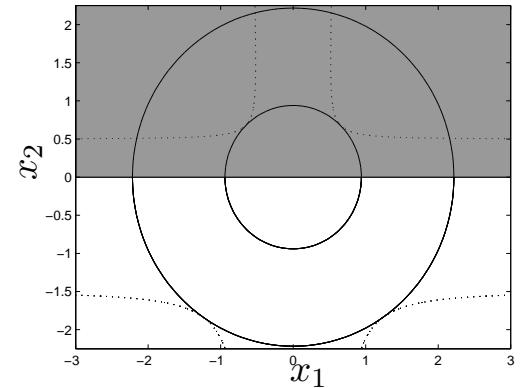

Figure 2: Example 4.1: $\mathcal{X}_{2}$ (white area), the curve $\Delta v_{2}(x)=0$ (dotted lines), the curve $\mathcal{V}\left(\hat{c}_{2}\right)$ for Problem A (inner solid line), the curve $\mathcal{V}\left(\hat{c}_{2}\right)$ for Problem B (outer solid line).

$\mathcal{X}_{2}=\mathbb{R}^{2} \backslash \mathcal{X}_{1}=\left\{x: x_{2}<0\right\}$

Figure 1 and Figure 2 show the largest estimates of the domain of attraction of individual subsystems $f_{1}(x)$ and $f_{2}(x), \mathcal{V}\left(\hat{c}_{1}\right)$ and $\mathcal{V}\left(\hat{c}_{2}\right)$ respectively. Regions $\mathcal{X}_{i}$ are shown as white area. By using Theorem 2, computational results show that $\hat{c}_{1}=0.4469$ and $\hat{c}_{2}=4.917$. Hence, $\hat{c}=\min \left\{\hat{c}_{1}, \hat{c}_{2}\right\}=0.4469$. As guaranteed by Theorem $2, \mathcal{V}(\hat{c})$ is a guaranteed inner estimate of $\mathcal{D}$.

\subsection{Example 2}

Consider a discrete-time nonlinear hybrid system in the form of (1) with

$f_{1}(x)=\left(\begin{array}{c}x_{2} \\ 0.6 x_{1}-x_{1} x_{2}\end{array}\right)$,

$f_{2}(x)=\left(\begin{array}{c}x_{2} \\ 0.2 x_{1}-0.2 x_{2}-x_{2}^{2}\end{array}\right)$,

with the function $v=2 x_{1}^{2}+x_{1} x_{2}+3 x_{2}^{2}$.

Let us consider Problem A (switched system). Figure 3 and Figure 4 show the largest estimates of the domain of attraction of individual subsystems $f_{1}(x)$ and $f_{2}(x), \mathcal{V}\left(\hat{c}_{1}\right)$ and $\mathcal{V}\left(\hat{c}_{2}\right)$ respectively. By using Theorem 


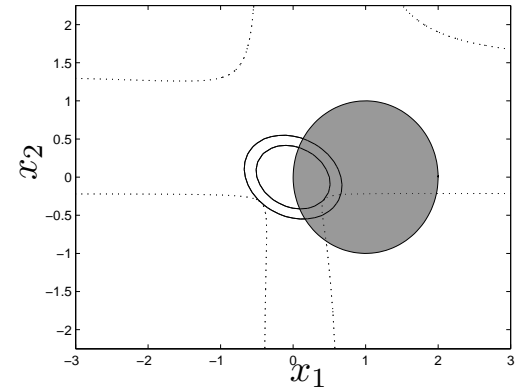

Figure 3: Example 4.2: $\mathcal{X}_{1}$ (white area), the curve $\Delta v_{1}(x)=0$ (dotted lines), the curve $\mathcal{V}\left(\hat{c}_{1}\right)$ for Problem A (inner solid line), the curve $\mathcal{V}\left(\hat{c}_{1}\right)$ for Problem B (outer solid line).

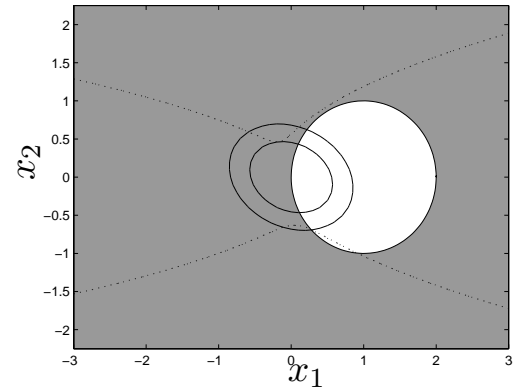

Figure 4: Example 4.2: $\mathcal{X}_{2}$ (white area), the curve $\Delta v_{2}(x)=0$ (dotted lines), the curve $\mathcal{V}\left(\hat{c}_{2}\right)$ for Problem A (inner solid line), the curve $\mathcal{V}\left(\hat{c}_{2}\right)$ for Problem B (outer solid line).

1, computational results show that $\hat{c}_{1}=0.5006$ and $\hat{c}_{2}=0.6309$. Hence, $\hat{c}=\min \left\{\hat{c}_{1}, \hat{c}_{2}\right\}=0.5006$. As guaranteed by Theorem $1, \mathcal{V}(\hat{c})$ is a guaranteed inner estimate of $\mathcal{D}$.

Next, let us consider Problem B (hybrid system). Consider $z_{1}(x)=$ $\left(x_{1}-1\right)^{2}+x_{2}^{2}-1$, we have

$\mathcal{X}_{1}=\left\{x: z_{1}(x) \geq 0\right\}$

$\mathcal{X}_{2}=\mathbb{R}^{2} \backslash \mathcal{X}_{1}=\left\{x:\left(x_{1}-1\right)^{2}+x_{2}^{2}-1<0\right\}$

Figure 3 and Figure 4 show the largest estimates of the domain of attraction of individual subsystems $f_{1}(x)$ and $f_{2}(x), \mathcal{V}\left(\hat{c}_{1}\right)$ and $\mathcal{V}\left(\hat{c}_{2}\right)$ respectively. Regions $\mathcal{X}_{i}$ are shown as white area. By using Theorem 2 , computational results show that $\hat{c}_{1}=0.8755$ and $\hat{c}_{2}=1.401$. Hence, $\hat{c}=\min \left\{\hat{c}_{1}, \hat{c}_{2}\right\}=0.8755$. As guaranteed by Theorem $2, \mathcal{V}(\hat{c})$ is a guaranteed inner estimate of $\mathcal{D}$.

Let us conclude this example by observing that the origin in this example cannot be a globally asymptotically stable equilibrium point since there exists another equilibrium point at $(-0.4,-0.4)^{\prime}$. In fact, Figure 5 and 6 shows the found estimates and an equilibrium point outside the true domain 
of attraction for both Problem A and B, and such a point is quite close to the found estimates.

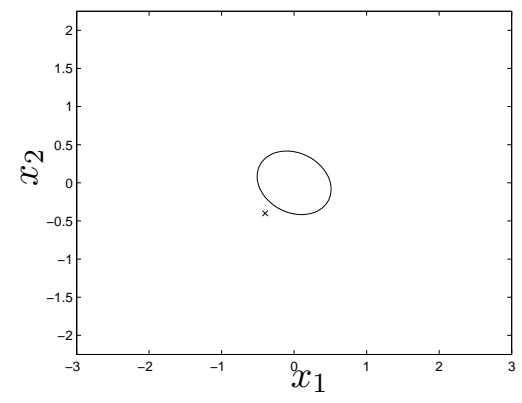

Figure 5: Example 4.2: the curve $\mathcal{V}(\hat{c})$ for Problem A (solid line), the equilibrium point $(-0.4,-0.4)^{\prime}$ (the X mark).

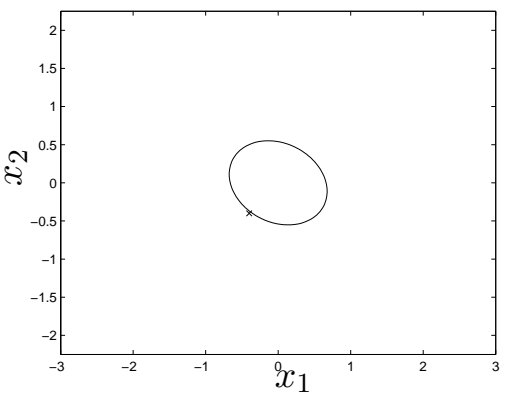

Figure 6: Example 4.2: the curve $\mathcal{V}(\hat{c})$ for Problem B (solid line), the equilibrium point $(-0.4,-0.4)^{\prime}$ (the X mark).

\section{Conclusion}

We have considered the estimation of the domain of attraction for discretetime switched and hybrid nonlinear systems. An approach based on convex programming has been proposed for computing a guaranteed inner estimate of the largest sublevel set of a Lyapunov function included in the domain of attraction. The conservatism of the provided estimate can be decreased by increasing the size of the optimization problem. Future work will investigate the possibility of searching for the Lyapunov function in the proposed approach.

\section{Acknowledgements}

The authors would like to thank the Editors and the Reviewers for their useful comments. This work is supported in part by the Research Grants Council of Hong Kong under Grant HKU711213E. 


\section{References}

[1] F. Amato, R. Ambrosino, G. De Tommasi, and A. Merola. Estimation of the domain of attraction for a class of hybrid systems. Nonlinear Analysis: Hybrid Systems, 5(3):212-219, 2011.

[2] M. S. Branicky. Introduction to hybrid systems. In D. Hristu-Varsakelis and W. S. Levine, editors, Handbook of Networked and Embedded Control Systems, pages 91-116. Birkhauser, 2005.

[3] G. Chesi. Estimating the domain of attraction for non-polynomial systems via LMI optimizations. Automatica, 45(6):1536-1541, 2009.

[4] G. Chesi. LMI techniques for optimization over polynomials in control: a survey. IEEE Transactions on Automatic Control, 55(11):2500-2510, 2010 .

[5] G. Chesi. Domain of Attraction: Analysis and Control via SOS Programming. Springer, 2011.

[6] G. Chesi. Rational Lyapunov functions for estimating and controlling the robust domain of attraction. Automatica, 49(4):1051-1057, 2013.

[7] G. Chesi, A. Garulli, A. Tesi, and A. Vicino. LMI-based computation of optimal quadratic Lyapunov functions for odd polynomial systems. International Journal of Robust and Nonlinear Control, 15(1):35-49, 2005.

[8] G. Chesi, A. Tesi, A. Vicino, and R. Genesio. On convexification of some minimum distance problems. In European Control Conference, Karlsruhe, Germany, 1999.

[9] R. Genesio, M. Tartaglia, and A. Vicino. On the estimation of asymptotic stability regions: State of the art and new proposals. IEEE Transactions on Automatic Control, 30:747-755, 1985.

[10] Z. Jarvis-Wloszek, R. Feeley, W. Tan, K. Sun, and A. Packard. Some control applications of sum of squares programming. In IEEE Conference on Decision and Control, pages 4676-4681, Maui, Hawaii, 2003.

[11] H. K. Khalil. Nonlinear Systems. Prentice Hall, 2001.

[12] D. Ludwig, B. Walker, and C. S. Holling. Sustainability, stability and resilience. Conservation Ecology, 1(1):7, 1997. 
[13] A. Merola, C. Cosentino, and F. Amato. An insight of tumor dormancy equilibrium via the analysis of its domain of attraction. Biomedical Signal Processing and Control, 3(3):212-219, 2008.

[14] S. Rozgonyi, K. M. Hangos, and G. Szederkenyi. Determining the domain of attraction of hybrid non-linear systems using maximal Lyapunov functions. Kybernetika, 46:19-37, 2010.

[15] Shakir Saat, Dan Huang, Sing K. Nguang, and A. H. Hamidon. Nonlinear state feedback control for a class of polynomial nonlinear discretetime systems with norm-bounded uncertainties: An integrator approach. Journal of the Franklin Institute, 350(7):1739-1752, September 2013.

[16] Shakir Saat, SingKiong Nguang, Chih-Min Lin, and Zahriladha Zakaria. Robust nonlinear $\mathrm{h}$ state feedback control of polynomial discrete-time systems: An integrator approach. Circuits, Systems, and Signal Processing, pages 1-16, 2013.

[17] J. F. Sturm. Using SeDuMi 1.02, a MATLAB toolbox for optimization over symmetric cones. Optimization Methods and Software, 11-12:625653, 1999.

[18] B. Tibken. Estimation of the domain of attraction for polynomial systems via LMI's. In IEEE Conference on Decision and Control, pages 3860-3864, Sydney, Australia, 2000.

[19] U. Topcu and A. Packard. Local stability analysis for uncertain nonlinear systems. IEEE Transactions on Automatic Control, 54(5):1042$1047,2009$.

[20] A. Vannelli and M. Vidyasagar. Maximal Lyapunov functions and domains of attraction for autonomous nonlinear systems. Automatica, 21(1):69-80, 1985. 